\title{
Úber den Einfluß der Nahrungszusammensetzung auf die Ausscheidung von 3-Methylhistidin und Kreatinin im Harn
}

\author{
Von M. Neuhäuser, U. Göttmann und K. H. Bäßler \\ Physiologisch-chemisches Institut, Johannes Gutenberg-Universität Mainz
}

(Eingegangen am 21. Mai/23. August 1984)

Zusammenfassung: Das von 7 gesunden Probanden mit einer eingewogenen Menge Fleisch aufgenommene 3-Methylhistidin wird quantitativ innerhalb von 2 Tagen ausgeschieden. Die gleichzeitig erfaßte KreatininAusscheidung bleibt bei 4 Probanden konstant, während 3 der Versuchsteilnehmer einen beträchtlichen Anstieg am Tag des Fleischkonsums verzeichnen. Bei der Stickstoffausscheidung zeigte sich in 5 von 7 Fällen eine Erhöhung der Werte in Abhängigkeit von der Fleischzufuhr.

\section{Dietary effects on the urinary excretion of 3-methylhistidine and creatinine}

Summary: 3-Methylhistidine in a defined amount of meat, consumed by 7 healthy persons is excreted quantitatively in the urine within 2 days. Simultaneously recorded creatinine excretion remained constant in 4 of the participants while in 3 cases a considerable increase was observed during the day of meat consumption. An increase in nitrogen excretion as a result of meat consumption was observed in 5 out of 7 persons.

\section{Einführung}

3-Methylhistidin kommt überwiegend in Muskelproteinen (Actin, Myosin) vor $(1,2)$. Beim Abbau von Muskelprotein freiwerdendes 3-Methylhistidin wird nicht reutilisiert und nimmt an weiteren biochemischen Umsetzungen im Organismus nicht teil (3), sondern wird quantitativ im Harn ausgeschieden (4, 5). Deshalb wird die Ausscheidung von 3-Methylhistidin als $\mathrm{Maß}$ für den Abbau von Muskelprotein angesehen und für Muskelproteinumsatzbestimmungen verwendet, wobei man fürr die Berechnung der Muskelmasse üblicherweise die Krèatinin-Ausscheidung heranzieht (6). Wẹsèntlich für die Aussagekraft bei diesem Verfahren ist die Erfassung des 3Methylhistidin mit endogenem Ursprung, was das Einhalten einer 3-Methylhistidin-freien, d. h. fleischfreien Diät erforderlich macht $(7,8)$, und woran viele Untersuchungen auf diesem Sektor scheitern. Intravenös verabreichtes 3-Methylhistidin wird quantitativ im Urin ausgeschieden (3) und bei einer 3-Methylhistidin-freien Diät sinkt die 3-MethylhistidinAusscheiderate innerhalb von 3-5 Tagen auf einen konstanten Wert herab, ohne mit signifikanten Veränderungen in der Kreatinin-Ausscheidung einherzugehen $(7,8)$.

Ziel dieser Studie war es zu untersuchen, wie sich die orale Zufuhr einer eingewogenen Menge Fleisch mit bekanntem 3-Methylhistidin-Gehalt in der 3-Methylhistidin- und Kreatinin-Ausscheidung widerspiegelt und ob somit auch unter - allerdings definiertem - Fleischkonsum Aussagen über den Muskelproteinstoffwechsel anhand von 3-Methylhistidinund Kreatinin-Bestimmungen im Urin möglich sind.

\section{Methodik}

Sieben gesunde Probanden, Institutsangehörige und Studenten, verzehrten am 5. Tag einer 6 Tage dauernden 3-Methylhistidinfreien Diät jeweils $250 \mathrm{~g}$ Tatar mit einem errechneten 3-Methylhistidin-Gehalt von $184,3 \mu \mathrm{mol}$. Zugrundegelegt wurden hierfür die Untersuchungen von Elia et al., die den 3-Methylhistidin-Gehalt von Rindfleisch mit $3,63 \mu \mathrm{moV} / \mathrm{g}$ fettfreies Trockengewicht bestimmten (9). Die Probanden wurden unter Anleitung einer Diätassistentin angehalten, ihren Proteinbedarf während der übrigen Tage durch andere Quellen als Muskelfleisch zu decken. 
Für die Bestimmung von 3-Methylhistidin im Urin wurde am 4., 5. und 6. Tag jeweils $24 \mathrm{~h}$-Urin gesammelt und parallel dazu die Kreatinin- sowic die Stickstoff-Ausschcidung gemessen.

3-Methylhistidin-Analysen wurden mittels Ionenaustauschchromatographic durchgeführt, wobei ein speziell hierfür entwickeltes Kurzprogramm angewendet wurde. Die technischen Details der Probenbereitung sowie des analytischen Verfahrens sind in einer früheren Arbeit ausführlich beschrieben (10). Die Kreatinin-Bestimmung erfolgte kinetisch nach einer modifizierten Methode auf der Grundlage der Jaffé-Reaktion (11). Gesamtstickstoff im Urin wurde durch eine Mikromethode nach Kjeldahl erfaßt (12).

Die tägliche Abbaurate für Muskelprotein erhält man aus dem Quotienten

3-Methylhistidinausscheidung [ $\mu \mathrm{mol} / \mathrm{d}]$

3-Methylhistidin in Muskelprotein [ $\mu \mathrm{mol} / \mathrm{g}]$

Der prozentuale Umsatz von Muskelprotein ergibt sich aus der Beziehung der Muskelproteinabbaurate zum gesamten Bestand an Muskelprotein : (13)

$$
\begin{aligned}
=100 & \times \frac{3-\text { Methylhistidinausscheidung }[\mu \mathrm{mol} / \mathrm{d}]}{\text { Kreatininausscheidung }[\mu \mathrm{mol} / \mathrm{d}]} \\
& \times \frac{1000}{3,63 \times 4 \times 113},
\end{aligned}
$$

wobei der durchschnittliche 3-Methylhistidin-Anteil beim Menschen 3,63 $\mu \mathrm{mol} / \mathrm{g}$ gemischtes Muskelprotein beträgt; der Faktor 4 geht aus der Annahme hervor, daß $1 \mathrm{~g}$ Kreatinin $20 \mathrm{~kg}$ Muskelmasse mit einem Proteinanteil von $20 \%$ entsprechen und 113 steht für das Molekulargewicht von Kreatinin.

\section{Ergebnisse}

Die Beschreibung der Versuchsteilnehmer sowie die Ausscheidung von 3-Methylhistidin, Kreatinin und Stickstoff am 4., 5. und 6. Tag finden sich in Tabelle 1.

Ausgangswerte bilden die Datén des 4. Tages der fleischfreien Diät. Sie betrugen im Mittel für die 3Methylhistidin-Ausscheidung $146 \pm 20 \mu \mathrm{mol} / \mathrm{d}$, für die Kreatinin-Ausscheidung 0,12 $\pm 0,02 \mathrm{mmol} / \mathrm{d}$ und für die Stickstoff-Ausscheidung 5,3 $\pm 3 \mathrm{~g} / \mathrm{d}$. Die an diesem Tag aus den genannten Daten errechnete Muskelproteinabbaurate wurde mit $40 \pm 5,6 \mathrm{~g} / \mathrm{d}$ und der prozentuale Umsatz von Muskelprotein mit $0,95 \pm 0,08 \%$ bestimmt. Am Tage des Fleischverzehrs stieg die durchschnittliche 3-MethylhistidinAusscheidung auf $298 \pm 25 \mu \mathrm{mol} / \mathrm{d}$ an. Die mittlere Kreatinin-Ausscheidung erhöhte sich um 19,8\% auf $0,14 \pm 0,01 \mathrm{mmol} / \mathrm{d}$ (nicht signifikant), während die ausgeschiedene Stickstoff-Menge mit 8,2 $\pm 1,14 \mathrm{~g} / \mathrm{d}$ signifikant $(p<0,05)$ erhöht war. Am Tag danach war die 3-Methylhistidin-Menge nur noch um $31 \pm$ $11,1 \mu \mathrm{mol} / \mathrm{d}$ im Vergleich zum Ausgangswert erhöht und betrug $177 \pm 20 \mu \mathrm{mol} / \mathrm{d}$. Gleichzeitig sank die Kreatininausscheidung auf $0,11 \pm 0,02 \mathrm{mmol} / \mathrm{d}$ her$a b$ und unterschied sich signifikant $(p<0,05)$ vom Wert des vorhergehenden Tages, an dem $250 \mathrm{~g} \mathrm{Ta}-$ tar verzehrt wurden, war aber nicht signifikant verschieden vom Ausgangswiwert. Die Stickstoff-Ausscheidung sank ebenfalls wieder ab und lag mit 6,9 \pm $1,1 \mathrm{~g} / \mathrm{d}$ zwischen den Werten des 4 . und 5. Tages.

Die Summe an 3-Methylhistidin, welches am 5. und 6. Tag über den Ausgangswiert hinaus ausgeschieden wurde, war im Mittel $183 \pm 28 \mu \mathrm{mol}$.

Tab. 1. Beschreibung der Versuchsteilnehmer sowie Ergebnisse für 3-Methylhistidin-, Kreatinin- und Stickstoff-Ausscheidung während

\begin{tabular}{|c|c|c|c|c|c|c|c|c|c|c|c|c|}
\hline \multirow[t]{2}{*}{ Nr. } & \multirow{2}{*}{$\begin{array}{l}\text { Ge- } \\
\text { schlecht }\end{array}$} & \multirow{2}{*}{$\begin{array}{l}\text { Alter } \\
\text { (Jahre) }\end{array}$} & \multirow{2}{*}{$\begin{array}{l}\text { Körper- } \\
\text { gewicht }\end{array}$} & \multicolumn{3}{|c|}{ Tag $4=$ fleischfrei } & \multicolumn{3}{|c|}{ Tag $5=250 \mathrm{~g}$ Tatar } & \multicolumn{3}{|c|}{ Tag $6=$ fleischfrei } \\
\hline & & & & $\begin{array}{l}\text { 3-Me- } \\
\text { thyl- } \\
\text { histidin } \\
\text { ( } \mu \mathrm{mol} / \mathrm{d})\end{array}$ & $\begin{array}{l}\begin{array}{l}\text { Krea- } \\
\text { tinin }\end{array} \\
(\mathrm{mmol} / \mathrm{d})\end{array}$ & $\begin{array}{l}\text { Stick- } \\
\text { stoff } \\
(\mathrm{g} / \mathrm{d})\end{array}$ & $\begin{array}{l}\text { 3-Me- } \\
\text { thyl- } \\
\text { histidin } \\
(\mu \mathrm{mol} / \mathrm{d})\end{array}$ & $\begin{array}{l}\text { Krea- } \\
\text { tinin } \\
(\mathrm{mmol} / \mathrm{d})\end{array}$ & $\begin{array}{l}\text { Stick } \\
\text { stoff } \\
(\mathrm{g} / \mathrm{d})\end{array}$ & $\begin{array}{l}\text { 3-Me- } \\
\text { thyl- } \\
\text { histidin } \\
(\mu \mathrm{mol} / \mathrm{d})\end{array}$ & $\begin{array}{l}\begin{array}{l}\text { Krea- } \\
\text { tinin }\end{array} \\
\text { (mmol/d) }\end{array}$ & $\begin{array}{l}\text { Stick- } \\
\text { stoff } \\
\text { (g/d) }\end{array}$ \\
\hline $\begin{array}{l}1 \\
2 \\
3 \\
4 \\
5 \\
6 \\
7\end{array}$ & $\begin{array}{l}q \\
q \\
q \\
q \\
q \\
q \\
q \\
0\end{array}$ & $\begin{array}{l}30 \\
21 \\
56 \\
27 \\
27 \\
26 \\
59\end{array}$ & $\begin{array}{l}54 \\
55 \\
61 \\
47 \\
51 \\
59 \\
71\end{array}$ & $\begin{array}{l}145 \\
119 \\
160 \\
120 \\
123 \\
105 \\
252\end{array}$ & $\begin{array}{l}0,11 \\
0,09 \\
0,11 \\
0,09 \\
0,12 \\
0,14 \\
0,20\end{array}$ & $\begin{array}{l}5,2 \\
4,2 \\
4,3 \\
3,9 \\
5,3 \\
7,8 \\
6,6\end{array}$ & $\begin{array}{l}308 \\
301 \\
227 \\
267 \\
259 \\
301 \\
421\end{array}$ & $\begin{array}{l}0,18 \\
0,14 \\
0,11 \\
0,13 \\
0,12 \\
0,13 \\
0,19\end{array}$ & $\begin{array}{r}5,1 \\
9,1 \\
4,0 \\
9,8 \\
9,1 \\
12,1 \\
8,2\end{array}$ & $\begin{array}{l}168 \\
216 \\
164 \\
123 \\
141 \\
160 \\
268\end{array}$ & $\begin{array}{l}0,11 \\
0,12 \\
0,08 \\
0,09 \\
0,08 \\
0,12 \\
0,19\end{array}$ & $\begin{array}{l}2,3 \\
6,5 \\
4,4 \\
9,4 \\
7,4 \\
9,7 \\
8,7\end{array}$ \\
\hline \multirow{2}{*}{\multicolumn{2}{|c|}{$\begin{array}{l}\text { Mittelwert } \\
\text { Standard- } \\
\text { abweichung }\end{array}$}} & 35,1 & 56,9 & 146 & 0,12 & 5,3 & 298 & 0,14 & 8,2 & 177 & 0,11 & 6,9 \\
\hline & & 6,3 & 3,2 & \pm 20 & $\pm 0,02$ & $\pm 0,34$ & \pm 25 & $\pm 0,01$ & $\pm 1,14$ & $\begin{array}{c} \pm 20 \\
::\end{array}$ & $\pm 0,02$ & $\pm 1,1$ \\
\hline
\end{tabular}
der Versuchstage 4, 5 und 6. 


\section{Diskussion}

Die vorliegende Studie hat ergeben, daß mit der Nahrung zugeführtes 3-Methylhistidin quantitativ innerhalb von 2 Tagen ausgeschieden wird.

Analysen zum 3-Methylhistidin-Gehalt in Proteinen aus unterschiedlichen Fleischquellen (Rind, Schwein, Geflügel, Fisch) ergaben eine bemerkenswerte Konstanz der Ergebnisse, die sich zwischen 3,56 und $3,78 \mu \mathrm{mol} / \mathrm{g}$ Protein (9) bewegen. Davon ausgehend, daß in $250 \mathrm{~g}$ Tatar $21 \%$ - also $52,5 \mathrm{~g}-$ Protein enthalten sind (14) und die die Basalwerte übersteigende 3-Methylhistidin-Ausscheidung am 5. und 6. Versuchstag $183 \mu \mathrm{mol}$ betrug, bedeutet eine erstaunliche Úbereinstimmung mit dem berechneten 3-Methylhistidin-Gehalt von $187 \mu \mathrm{mol}$. Ein Anteil von $83,1 \%$ der mit der Nahrung zugeführten 3-Methylhistidin-Menge wurden am Tag des Konsums ausgeschieden, während die restlichen $16,9 \%$ am darauffolgenden Tag im Urin gefunden wurden, wobei die mittlere 3-Methylhistidin-Ausscheidung schon nicht mehr signifikant vom Ausgangswert verschieden war.

Da die absolute tägliche 3-Methylhistidin-Ausscheiderate von der Muskelmasse abhängig ist (15), wird - zur besseren UUbersicht bei Untersuchungen von sehr heterogenen Personengruppen - die 3-Methylhistidin-Ausscheidung zur Kreatininausscheidung in Beziehung gesetzt oder der Muskelproteinumsatz angegeben, wobei der tägliche Muskelproteinabbau zur gesamten Muskelmạsse in Beziehung gesetzt wird. Eigene Untersuchungen haben ergeben, daß diese beiden Angaben unter normalen Bedingungen unabhängig von Geschlecht und Muskelmasse sind (16). Die Ausgangswerte der Versuchspersonen für die Muskelproteinabbaurate und der prozentuale Umsatz von Muskelprotein $(15,17)$ liegen in dem in der Literatur beschriebenen Referenzbereich. Da auch die Berechnung der Muskelmasse üblicherwei- se anhand der Kreatinin-Ausscheidung erfolgt, setzt dies voraus, daß die Kreatinin-Ausscheidung eine konstante und von der Diät unabhängige Größe ist. Hierüber existiert zum heutigen Zeitpunkt keine einheitliche Meinung (18). Obwohl der mittlere Anstieg von $19 \%$ am Tag des Fleischverzehrs nicht signifikant ist, zeigen einige Versuchsteilnehmer (Nr. 1, 2 und 4) erhebliche Erhöhungen bei der Kreatinin-Ausscheidung. Auch der signifikante Abfall in der Kreatininausscheidung am folgenden Tag läßt Zweifel an der Aussagekraft der Kreatinin-Ausscheidung unter Fleischkonsum in bezug auf die Muskelmasse aufkommen. Hierzu müßte man auf die Basalwerte zurückkommen.

Die Stickstoff-Ausscheidung zeigt am 4. und 6. Tag relativ niedrige Werte und am Tag, an dem Fleisch konsumiert wurde, wurde ein signifikanter Anstieg registriert. Hierfür dürfte die höhere Proteinzufuhr nach der fleischfreien Ernährungsperiode verantwortlich sein.

Zusammenfassend läßt sich sagen, daß Untersuchungen zum Muskelproteinstoffwechsel anhand der 3-Methylhistidin-Ausscheidung unter einer fleischhaltigen Diät zwar möglich sind, Voraussetzung hierfür aber die kontante Zufuhr einer abgewogenen Menge Fleisch ist. Unter solchen Bedingungen dürften relative Veränderungen in der 3-MethylhistidinAusscheidung durchaus sichtbar werden. Auch quantitative Aussagen sind nach diesen Ergebnissen möglich, wenn man die exogen zugeführte 3-Methylhistidin-Menge von der Gesamtausscheidung subtrahiert. Umgekehrt ist es möglich, anhand der 3-Methylhistidin-Ausscheidung Aufschlüsse über den Fleischkonsum zu erhalten. Die Kreatinin-Ausscheidung als Bezugsgröße bei vielen Untersuchungen ist mit Vorsicht anzuwenden, da sie offensichtlich nicht bei allen Menschen unabhängig von der Ernährungsweise ist.

\section{Literatur}

1. Haverberg, L. N., Omstedt, P. T., Munro, H. N. \& Young, V. R. (1975) Biochim. Biophys. Acta 405, 67-71.

2. Elia, M., Carter, A. \& Smith, R. (1979) Brit. J. Nutr. 42, 567-570.

3. Long, C. L., Haverberg, L. N., Young, V. R., Kinney, J. M., Munro, H. N. \& Geiger, J. W. (1975) Metabolism 24, 929935.

4. Young, V. R., Alexis, S. O., Baliga, B. S. \& Munro, H. N. (1972) J. Biol. Chem. 247, 3592-3600.

5. Young, V. R., Baliga, B. S., Alexis, S. O. \& Munro, H. N. (1970) Biochim. Biophys. Acta 199, 297-300.

6. Graystone, J. E. (1968) in: Human Growth (Cheek, D. B., ed.) pp. 183-197, Lea \& Febiger, Philadelphia.

7. Tomas, F. M., Ballard, F. J. \& Pope, L. M. (1979) Clin. Sci. $56,341-346$.

8. McKeran, R. O., Halliday, D. \& Purkiss, P. (1978) Clin. Sci. Mol. Med. 54, 471-475.

9. Elia, M., Carter, A., Bacon, S. \& Smith, R. (1980) Clin. Sci. $59,509-511$.

10. Neuhäuser, M. \& Fürst, P. (1979) Analyt. Biochem. 92, 294-304.

11. Jaffé, M. (1886) Zeitschr. Physiol. Chemie 10, 391-400.

12. Henry, R. J. (1974) in: Clinical Chemistry, Principles and Techniques (Cannon, D. C. \& Winkelman, J. W., eds.), 2nd Ed., pp. 409-411, Harper \& Row Publishers Hagerstown, Maryland. 
13. Ballard, F. J. \& Tomas, F. M. (1983) Clin. Sci. 65, 209-215.

14. Wirths, W. (1982) Kleine Nährwerttabelle der Deutschen Gesellschaft für Ernährung e.V., Umschau Verlag, Frankfurt.

15. Lukaski, H. C., Mendez, J., Buskirk, E. R. \& Cohn, S. H. (1981) Am. J. Physiol. 240, E302-E307.
16. Neuhäuser, M. \& Bäßler, K. H. (1984) Z. Ernährungswiss. 23, 171-180.

17. Bilmazes, C., Uauy, R., Haverberg, L. N., Munro, H. N. \& Young, V. R. (1978) Metạbolism 27, 525-530.

18. Wood, C., Schneeman, P., Zezulka, A., Calloway, D. H. \& Margen, S. (1976) Fed. Proc. (Abstracts) 35, Abstract no. 1609.

1

Dr. Monika Neuhäuser

Physiologisch-chemisches Institut

Saarstraße 21

D-6500 Mainz 